\title{
Recognition Method for Abnormal Water Surface Based on SOM
}

\author{
Jianjun Zuo \\ School of Sciences, Guizhou University of Engineering \\ Science; Bijie Guizhou 551700, China \\ E-mail:jj_zuo@163.com
}

\begin{abstract}
Abnormal Water Surface monitoring and recognition technique still is the research focus at present. In this paper, we first establish a background model with Gaussian Mixture Model, achieve the abnormal region quickly using background difference method, then, a improve algorithm of color moment calculation is used to extract the abnormal features, finally the SOM model is used to cluster the characteristics of the data, the data classifier is established.
\end{abstract}

Keywords-Background subtraction; Color moments; Color histogram; Clustering; Recognition.

\section{INTRODUCTION}

Among the environmental protection, the intelligent detection of rivers, reservoirs and lakes has become the focal point. It has aroused board attention in academy and industry, because of its broad applications and commercial value. Since the late 1980s, the video surveillance system is established by the United States, Japan, Western Europe and other countries. Without human intervention, the image sequence from visual sensor were analysed, through computer image processing, the positioning and recognition of the target motion in dynamic scenes is implemented, on the basis of this system, it is raising the signal about the automatic dredging, and start the equipment, the automatic cleaning up to debris.Effective clustering and accurate recognition is a crucial step of the video surveillance system, clustering analysis is a division of data into similarity groups according to given rules. Clustering analysis is a non supervised machine learning method and it has been applied in many fields [1]. Classical clustering algorithms are Kmeans, CURE, the BP neural network, etc, class number for clustering a dataset is the precondition of the classical algorithm,it based on experience more, and requires repeated attempts to achieve, on the whole, it reduced the efficiency of clustering and the credibility of the result.

SOM was proposed by the dcholars Kohonen firstly in 1981 and it is A special neural network algorithm [2]. SOM can project high-dimensional data on a low-dimensional regular grid,thus in order to achieve the purpose of dimensionality reduction.SOM displays the superiority in dealing with clustering problems,compared with other methods, it has several advantages such as .self-learning, network stability, clustering process without external evaluation function is given and be able to identify the characteristics of the vector space etc,therefore, the model has wide applications to the pattern recognition, signal processing, Vector quantization and soon $[3,4,5]$.

In this paper, the main idea is firstly to segmentation the image by background difference method algorithm; Then, a

\author{
Youfu Wu \\ Zunyi Normal College; Zunyi Guizhou 563002, China \\ E-mail: youfu_wu_64@126.com
}

algorithm of color moment calculation is used to extract the abnormal features. Second, then the SOM is used to cluster the data containing all kinds of abnormal, to establish abnormal classifier. Finally a abnormal classifier was used in recognition.

\section{SEGMENTATION FLOATING AREA}

\section{A. Background model estimation}

There are floater senarios where it is varies with the weather, day and night, then resulting in the change of floater background.

In order to segment floater area exactly,we built the non-pulluted water background based on adaptive Gaussian mixture model.It can be used to detect and segment floater.We close to model the recent history of each pixel, $\left\{I_{1}, I_{2}, \cdots I_{t}\right\}$, as a mixture of $K$ Gaussian distributions. The probability of observing the current pixel value is [6]

$$
P(I(x, y, t))=\sum_{i=1}^{K} \omega_{i, t} \eta_{i}\left(I, \mu_{i, t}, \sigma_{i, t}\right)
$$

Where $\mathrm{K}$ is the number of distributions, $\omega_{i, t}$ is an estimate of the weight of ith Gaussian in the mixture at time $\mathrm{t}$, where

$$
\sum_{i=1}^{K} \omega_{i, t}=1
$$

$\mu_{i, t}$ and $\sigma_{i, t}$ are the mean value and covariance matrix of the ith Gaussian in the mixture at the t,and where $\eta_{i}\left(I, \mu_{i, t}, \sigma_{i, t}\right)$ is a Gaussian probability density function

$$
\eta_{i}\left(I, \mu_{i, t}, \sigma_{i, t}\right)=\frac{1}{\sqrt{(2 \pi)^{n}\left|\sigma_{i, t}\right|}} \exp \left(\frac{1}{2 \sigma_{i, t}}\left(I-\mu_{i, t}\right)^{T}\left(I-\mu_{i, t}\right)\right)
$$

\section{B. Object area segmentation}

In the floating area appears, the current image is different from the background image in the gray-level value. Then,the current image was subtracted from the background image and the floater is detected [7]. We have:

$$
I_{s}=\left|I_{n}-I_{b g}\right|
$$

For a fixed point $(x, y)$, if the $I_{s}$ is larger than the threshold value T,then $(x, y)$ belongs to floating area. Due to the influence of the noise in nature environment, $I_{s}$ can't 
represent precisely the object and background,it is necessary to segment. Threshold the result image then get the binary image. The best threshold value of result image with the method of the biggest proportion of square deviations is calculated. This is, the optimal threshold can be derived from $\delta_{\text {inter }}^{2}=P_{1} P_{2}\left(m_{1}-m_{2}\right)^{2} \quad[8,9,10]$,

Where

$$
m_{1}=\sum_{i=0}^{k} \frac{i h_{i}}{P_{1}}, P_{1}=\sum_{i=0}^{k} h_{i} ; m_{2}=\sum_{i=k+1}^{K} \frac{i h_{i}}{P_{2}}, P_{2}=\sum_{i=k+1}^{K} h_{i}
$$

\section{EXTRACTING OBJECT FEATURES}

The color features is basing on the pixels, the surface properties of the scene corresponding to the image were described in this paper. Because the color is robust to small change of the direction and size of the image, also, we took the color feature to describe the floater. This paper uses color moment, the improved color moment and reducing color histogram, and it's used to describe the features of the floater.

\section{A. Color moment}

The color moment is a method of the simple and effective [11]. Any of the color distribution in the image can be represented by their moment. In addition, because color information is a primary lower-order moment. Therefore, we are generated which represents the distribution of hue values in an image with the 1st, 2nd and 3rd order moment. To properly using the color, to accurately extract floating feature, Therefore, we select for use RGB model, statistical color moments of the R, G, B channel respectively, the color moments in the ith color channel was defined as follows:

$$
\mu_{i}=\frac{1}{N} \sum_{j=1}^{N} f_{i j} \sigma_{i}=\sqrt{\frac{1}{N} \sum_{j=1}^{N}\left(f_{i j}-\mu_{i}\right)^{2}}, \quad \xi_{i}=\sqrt[3]{\frac{1}{N} \sum_{j=1}^{N}\left(f_{i j}-\mu_{i}\right)^{3}}
$$

Where $f_{i j}$ is the jth pixel values in the ith color channel, $N$ is number of pixels in the image. So, for a sample image, it will be able represented by 9 dimensional vectors base on the color moment of R,G,B component. It was defined as follows:

$$
E_{k}=\left(\mu_{k 1}, \sigma_{k 1}, \xi_{k 1}, \mu_{k 2}, \sigma_{k 2}, \xi_{k 2}, \mu_{k 3}, \sigma_{k 3}, \xi_{k 3}\right)
$$

\section{B. Improved color moment}

In color moment, the 3rd order moment can cause offset each other, so as to weakes the floater color information, therefore the 3rd order moments is replace by the 3rd order absolute moments. Improved the color moments was defined as follows:

$$
\mu_{i}=\frac{1}{N} \sum_{j=1}^{N} f_{i j} \quad \sigma_{i}=\sqrt{\frac{1}{N} \sum_{j=1}^{N}\left(f_{i j}-\mu_{i}\right)^{2}}
$$

$$
\bar{\xi}_{i}=\sqrt[3]{\frac{1}{N} \sum_{j=1}^{N}\left|f_{i j}-\mu_{i}\right|^{3}}
$$

At this point, the features of the sample figure was defined as follows:

$$
\bar{E}_{k}=\left(\mu_{k 1}, \sigma_{k 1}, \bar{\xi}_{k 1}, \mu_{k 2}, \sigma_{k 2}, \bar{\xi}_{k 2}, \mu_{k 3}, \sigma_{k 3}, \bar{\xi}_{k 3}\right)
$$

\section{Color histogram feature}

\section{1) Color histogram}

The color histogram is on a given space to statistics the number of each color component [12]. And it can also obtain distribution of all sorts of color component. Then the color value as the abscissa, the proportional distribution as the ordinate, it was called the color histogram of the image. The color histogram of the image $\mathrm{H}$ was defined as follows:

$$
H=\left\{h\left[c_{1}\right], h\left[c_{2}\right], \cdots, h\left[c_{k}\right], \cdots, h\left[c_{n}\right] \mid 0 \leq h\left[c_{k}\right] \leq 1\right\}
$$

$c_{k}$ is the kth color, $h\left[c_{k}\right]$ is the frequency of the ith colors. It was defined as follows:

$$
h\left[c_{k}\right]=\frac{\sum_{i=0}^{M-1} \sum_{j=0}^{N-1} f(i, j)}{M \times N}
$$

Where, $f(i, j)=\left\{\begin{array}{ll}1 & I(i, j)=c_{k} \\ 0 & I(i, j) \neq c_{k}\end{array}\right.$, the $\mathrm{M}$ and $\mathrm{N}$ was the width and height of the image.

2) Simplify the color histogram

For the RGB color space, there are $256 \times 256 \times 256=1.68 \times 10^{7}$ colors, the color space for true color space. If the relative calculations, the algorithm has high algorithmic complexity. Therefore, it is essential to the simplification of the histogram. We discusses the new color space by uniform compression method to determine, True color space is compressed to $16 \times 16 \times 16=4096$ color area, the color histogram is:

$$
\bar{H}=\left\{\bar{h}\left[c_{1}\right], \bar{h}\left[c_{2}\right], \cdots, \bar{h}\left[c_{k}\right], \cdots \bar{h}\left[c_{n}\right] \mid 0 \leq \bar{h}\left[c_{k}\right] \leq 1\right\}
$$

Where, $c_{k}$ is the kth color area, $\bar{h}\left[c_{k}\right]$ is the frequency of the ith colors area, $n$ is the number of the color area. $\bar{h}\left[c_{k}\right]$ was defined as follows:

$$
\bar{h}\left[c_{k}\right]=\frac{\sum_{i=0}^{M-1} \sum_{j=0}^{N-1} f(i, j)}{M \times N}
$$


Where, $f(i, j)=\left\{\begin{array}{l}1 I(i, j) \in C_{k} \\ 0 I(i, j) \notin C_{k}\end{array}\right.$, the $\mathrm{M}$ and $\mathrm{N}$ was the width and height of the image.

3) Method of reducing dimensional of histogram feature base on PCA

The dimensionality of the color histogram is too high $(1 \times 4096)$, the computation amount is large, But as you have seen that many element value is zero. The histogram is a sparse matrix, Mainly consists of noise and redundant. Therefore, it is necessary to reduce the dimension of the color histogram. Dimensionality of input variables are reduced using Principal Component Analysis (PCA) [13].

Let the color histogram consists of $n$ frequency, it is the formation of $n$ dimensional row vectors $x_{i}=\left(x_{i 1}, x_{i 2}, \cdots, x_{i n}\right)$.A sample set is formed by the color histogram of $\mathrm{m}$ the floater images. the matrix as following:

$X=\left(x_{1}, x_{2}, \cdots, x_{m}\right)^{T} \in R^{m \times n}$. Let $\psi$ is all the sample's average vector,

$A=\left(x_{1}-\psi, x_{2}-\psi, \cdots, x_{m}-\psi\right)^{T} \quad$ can be gained by subtracting $\psi$ vector from each row of $X$ vector. Finally, calculating feature vector $\lambda_{i}$ of $A^{T} A$, Feature subspace $P \in R^{n \times k}$ is formed by $\mathrm{K}$ the biggest feature vectors. It can project $X$ to lower one:

$$
T=A P
$$

Where, $T \in R^{m \times K}$

\section{THE FLOATER RECOGNITION BASE ON SOM}

In this paper, the SOM method is used for the recognition of the floater, First, the features are extracted from different floater images, SOM neural network was trained and set up the floater classifiers. Second, the features are extracted from segmentation floaters on the water, its classification is estimated by compared with class-center of the floater classifiers.

\section{A. Classifying float objects based on SOM}

\section{1) Network structure design}

$\mathrm{K}$ features are extracted from the floater images, the experience of artificial recognition indicate that according to the color difference, may divede into 10 kinds. In the clustering, the implement 50 floater images feature extraction, So the output layer is set to 100 neurons of two-dimensional structure, each floater feature individually associates with a winning neuron, after the training, keep neurons together that belong together.

2) Weights initialization

Before the training, each neurons weight $w_{j}$, so you need to initialize, The common practice is to each weight vector of the output laye is initialized by the smaller the random number, and weight vector normalization. In this paper, the samples mainly focuses on a local area of the two-dimensional space. To choose $\mathrm{N}$ input samples with randomly from $m$ sample model, as the initial value, that is

$$
W_{j}(0)=X^{k_{r a m}}, j=1,2, \cdots, N
$$

Where, $k_{\text {ram }}$ is random numbers of input samples, $k_{\text {ram }} \in\{1,2, \cdots, m\}$.

3) Network parameter design

The size of the winner areas $N_{j^{*}}(t)$ are expressed as area radius $r(t), r(t)$ was define as:

$$
r(t)=\mathrm{C}_{1}\left(1-\frac{t}{t_{m}}\right)
$$

Where, $C_{1}$ is normal number of number of input layer neurons, $t_{m}$ is max training time, $\eta(t)$ is learning rate of t moment. It was define as follows:

$$
\eta(t)=C_{2} \exp \left(-B_{2} t / t_{m}\right)
$$

Where, $C_{2}$ is constant between 0 and $1, B_{2}$ is constant more than 1.

\section{B. Target recognition based on SOM}

To detect the floater, the size is same as sample, to txtract the feature, and then put the feature vectors into the SOM sorter to recognize. The result is a point $(x, y)$ on the two-dimensional space, then calculate the Euclidean distance with the center of clustering could be gained by using the method of clustering, the calculating method of Euclid distance was as follows:

$$
d_{j}=\sqrt{\left(x-O_{j}\right)^{2}+\left(y-O_{j}\right)^{2}} \quad(j=1,2, \cdots, P)
$$

Select the minimum distance node $j^{*}$, that is:

$$
d^{*}=d_{j^{*}}=\min \left(d_{j}\right) \quad(j=1,2, \cdots, P)
$$

\section{EXPERIMENTAL RESULTS AND COMPARISON}

In this section, in order to test the validity of the proposed approach, experimental result on real data sets are here given.

In our experiments, we take the images of different polluted reservoir and rivers of Guiyang as experimental

\begin{tabular}{|c|c|c|c|}
\hline Floater sample & Classification & Floater sample & Classification \\
\hline & 1 & 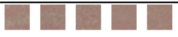 & 6 \\
\hline DEDD & 2 & 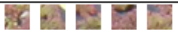 & 7 \\
\hline 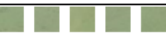 & 3 & 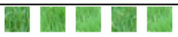 & 8 \\
\hline 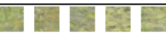 & 4 & SW & 9 \\
\hline 20 & 5 & $A x \times B A$ & 10 \\
\hline
\end{tabular}
source; and programmer with Matlab for test.

From each of the floater image, we have selected 5 categories, a total of 50 categories images of $32 * 32$ as training sample. An experiment result was shown in table 1. TABLE 1 FLOATER SAMPLE IMAGE AND CATEGORY NUMBER 
The color moment and improved color moment and color histogram for each floater sample are computed. The sample characteristics as input vector of SOM. Output layer has $10 * 10=100$ neurons, the time of training are 20000 times. Figure 1 is clustering simulation of color moment; Figure 2 is clustering simulation of improved color moment; Figure 3 is clustering simulation of color histogram. TABLE 2 is time statistics.

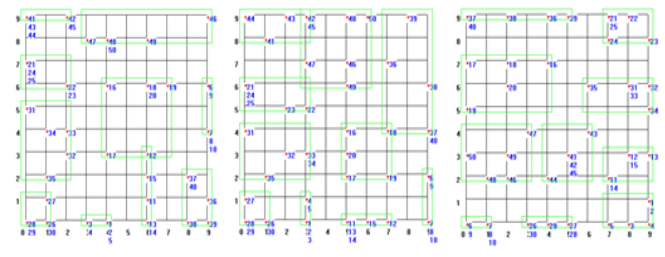

Figure 1 color moment Figure 2 improved color moment Figure 3 color histogram TABLE 2 time statistics (training 20000 times)

\begin{tabular}{|c|c|c|c|}
\hline Method & $\begin{array}{c}\text { Color } \\
\text { histogram }\end{array}$ & $\begin{array}{c}\text { Color } \\
\text { moment }\end{array}$ & $\begin{array}{c}\text { Improved } \\
\text { color moment }\end{array}$ \\
\hline $\begin{array}{l}\text { The clustering } \\
\text { of time }\end{array}$ & $24.1 \mathrm{~s}$ & $27.5 \mathrm{~s}$ & $27.5 \mathrm{~s}$ \\
\hline
\end{tabular}

For testing the effect of the target recognition, we have selected 100 categories from floater images randomly, as testing sample. TABLE 3 is the comparison of recognition results. TABLE 4 is Recognition rate TABLE 3 RECOGNITION RESULT COMPARED (training 20000 times)

\begin{tabular}{|c|c|}
\hline & ara \\
\hline & $\begin{array}{lllllllllllllllllll}4 & 4 & 4 & 4 & 4 & 4 & 4 & 4 & 4 & 2 & 2 & 2 & 2 & 2 & 2 & 2 & 2 & 2 & 2 \\
\end{array}$ \\
\hline & 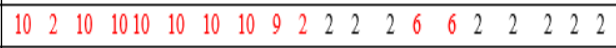 \\
\hline & $\begin{array}{lllllllllllllll}10 & 10 & 410 & 10 & 10 & 4 & 4 & 4 & 2 & 2 & 2 & 2 & 2 & 2 & 2\end{array}$ \\
\hline & $\begin{array}{llllllllllllllll}4 & 4 & 4 & 8 & 4 & 4 & 10 & 4 & 4 & 4 & 2 & 2 & 2 & 2 & 2 & 2\end{array}$ \\
\hline & 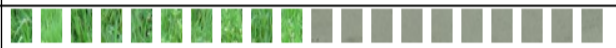 \\
\hline & 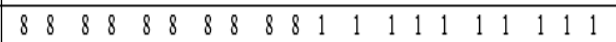 \\
\hline & $\begin{array}{cccccccccccccccccccc}5 & 8 & 5 & 5 & 5 & 8 & 8 & 8 & 5 & 8 & 1 & 1 & 1 & 1 & 1 & 1 & 1 & 1 & 1 & 1\end{array}$ \\
\hline & 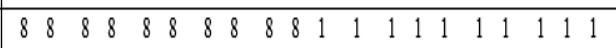 \\
\hline & 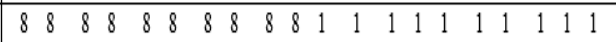 \\
\hline & 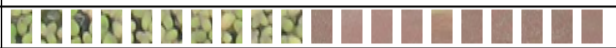 \\
\hline & $0 \begin{array}{llllllllllllllll}10 & 10 & 10 & 1010 & 10 & 1010 & 10 & 6 & 6 & 6 & 6 & 6 & 6 & 6 & 6 & 6\end{array}$ \\
\hline & 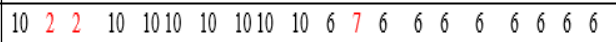 \\
\hline & 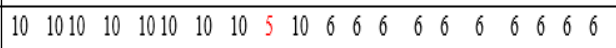 \\
\hline & 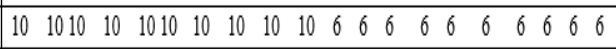 \\
\hline & 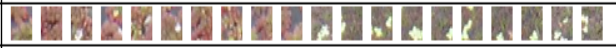 \\
\hline & 77777777775555555555 \\
\hline & 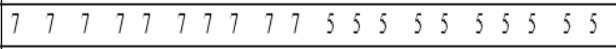 \\
\hline & 77777777777755555555555 \\
\hline & $\begin{array}{llllllllllllllllll}7 & 7 & 7 & 7 & 7 & 777 & 77 & 5 & 5 & 5 & 5 & 5 & 5 & 5 & 5 & 5 & 5\end{array}$ \\
\hline & 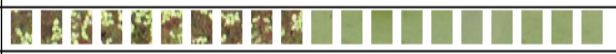 \\
\hline & $\begin{array}{llllllllllllllllllll}9 & 9 & 9 & 9 & 9 & 9 & 9 & 9 & 9 & 9 & 3 & 3 & 3 & 3 & 3 & 3 & 3 & 3 & 3 & 3\end{array}$ \\
\hline & $\begin{array}{lllllllllllllllllllllllll}9 & 9 & 9 & 9 & 9 & 9 & 9 & 9 & 9 & 9 & 3 & 3 & 6 & 3 & 3 & 3 & 3 & 3 & 10 & 3\end{array}$ \\
\hline & $\begin{array}{lllllllllllllllllllll}9 & 9 & 9 & 9 & 9 & 9 & 9 & 9 & 9 & 9 & 4 & 4 & 4 & 4 & 3 & 3 & 3 & 3 & 3 & 3 \\
\end{array}$ \\
\hline & $\begin{array}{lllllllllllllllllllll}9 & 9 & 9 & 9 & 9 & 9 & 9 & 9 & 9 & 9 & 3 & 3 & 4 & 3 & 3 & 3 & 3 & 3 & 3 & 3\end{array}$ \\
\hline
\end{tabular}

TABLE 4 RECOGNITION RATE COMPARED

\begin{tabular}{|l|c|c|c|c|}
\hline \multicolumn{1}{|c|}{ Method } & $\begin{array}{c}\text { Recognition rate } \\
(10000 \text { times })\end{array}$ & $\begin{array}{c}\text { Recognition rate } \\
(20000 \text { times })\end{array}$ & $\begin{array}{c}\text { Recognition rate } \\
(30000 \text { times })\end{array}$ & $\begin{array}{c}\text { Recognition rate } \\
\text { (40000 times) }\end{array}$ \\
\hline $\begin{array}{l}\text { Color } \\
\text { histogram }\end{array}$ & $82 \%$ & $78 \%$ & $77 \%$ & $81 \%$ \\
\hline $\begin{array}{l}\text { Color } \\
\text { moment }\end{array}$ & $94 \%$ & $90 \%$ & $92 \%$ & $91 \%$ \\
\hline $\begin{array}{l}\text { Improved color } \\
\text { moment }\end{array}$ & $90 \%$ & $97 \%$ & $97 \%$ & $97 \%$ \\
\hline
\end{tabular}

\section{CONCLUSIONS AND FUTURE WORK}

In this paper, we extracted the feature variables in the RGB color space. To deal with the challenges of abnormal water surface monitoring, the SOM is cited to distinguish abnormal object from normal ones. In our future work, we shall address the problem of the information fusion.

\section{ACKNOWLEDGMENT}

This work was supported by Science Technique Department of Guizhou Province of China (no. [2013]2146; no.LH[2014]7533) and Supported by Guizhou Education Department (no.KY[2014]275).

\section{REFERENCES}

[1] Han J,Kamber M.Data Mining:Concepts and Techniques.2nd ed [M]. San Francisco: Morgan Kaufmann Publishers, 2006.

[2] Kohonen T. Self-Organizing Maps [M]. Berlin: Springer Verlag, 2001.

[3] Mu Chun Su, Hsiao Te Chang. A new model of self-organizing neural network and its application in data projection [J]. IEEE Transactions on Neural Networks, 2001, 12(1):153-162.

[4] Rahul Singh, Vladimir Cherkassky, Nikolaos Papanikolopoulos. Self-organizing maps for the Skeletonization of spars shapes [J]. IEEE Transactions on Neural Networks, 2000, 11(1): 241-250.

[5] Hujun Yin, Nigel MAllinson. Self organizing mixture networks for probability density estimation [J]. IEEE Transactions on Neural Networks, 2001, 12(2): 405-409.

[6] Stauffer C, Grimson W. Learning pattern of activity using real-time tracking [J]. IEEE Transactions on Pattern Analysis and Machine Intelligence, 2000, 22(8): 747-757.

[7] Y.Wu, J.Shen and M.Dai. Traffic object detections and its action analysis [C]. International journal of pattern recognition letter (Vol.26,No.13(2005.10), PP1963-1984.

[8] Y.Wu and J.Shen. Moving object detection using orthogonal Gaussian-Hermite moments [C], Visual communication and image processing, 2004, Proc SPIE-IS \& T electronic imaging SPIE. Vol.5308, USA, p841-849, 2004.

[9] Y.Wu and G.Zhou, Adaptive Human Body Tracking and Its Abnormality Deportment Analysis [C], ICNC'09-FSKD'09, 2009, China.

[10] Gang Zhou and Youfu Wu. Anomalous Event Detection Based on Self-Organizing Map for Supermarket Monitoring. 2009 International Conference on Information Engineering and Computer Science (ICIECS2009), vol.1, pp.106-109, Wuhan,China, Dec,2009.

[11] Stricker M, Orengo M. Similarity of color images. IS\&T/SPIEC for Storage and Retrieval for Image and Video Databases III 1995.

[12] M J Swain, D H Ballar, Color indexing [J]. International Journal of Computer Vision, 1991, 7(1):11-32.

[13] Jonathon Shlens. "A Tutorial on Principal Component Analysis" 